\title{
Determinant and Inverse of Matrices of Real Elements
}

\author{
Nobuyuki Tamura \\ Shinshu University \\ Nagano, Japan
}

\author{
Yatsuka Nakamura \\ Shinshu University \\ Nagano, Japan
}

\begin{abstract}
Summary. In this paper the classic theory of matrices of real elements (see e.g. [12], [13]) is developed. We prove selected equations that have been proved previously for matrices of field elements. Similarly, we introduce in this special context the determinant of a matrix, the identity and zero matrices, and the inverse matrix. The new concept discussed in the case of matrices of real numbers is the property of matrices as operators acting on finite sequences of real numbers from both sides. The relations of invertibility of matrices and the "onto" property of matrices as operators are discussed.
\end{abstract}

MML identifier: MATRIXR2, version: 7.8.05 4.87.985

The articles [24], [30], [9], [2], [22], [31], [7], [4], [5], [8], [3], [6], [28], [26], [21], [14], [29], [32], [23], [25], [27], [15], [34], [33], [19], [16], [11], [18], [20], [10], [17], [1], and [35] provide the terminology and notation for this paper.

\section{Preliminaries}

We use the following convention: $D$ denotes a non empty set, $k, n, m, i, j$, $l$ denote elements of $\mathbb{N}$, and $K$ denotes a field.

We now state several propositions:

(1) For all finite sequences $x, y$ of elements of $\mathbb{R}$ such that len $x=$ len $y$ and $x+y=\langle\underbrace{0, \ldots, 0}_{\operatorname{len} x}\rangle$ holds $x=-y$ and $y=-x$.

(2) Let $A$ be a matrix over $D$ and $p$ be a finite sequence of elements of $D$. If $p=A(i)$ and $1 \leq i$ and $i \leq \operatorname{len} A$ and $1 \leq j$ and $j \leq$ width $A$ and len $p=$ width $A$, then $A_{i, j}=p(j)$. 
(3) Let $a$ be a real number and $A$ be a matrix over $\mathbb{R}$. Suppose len $(a \cdot A)=$ len $A$ and $\operatorname{width}(a \cdot A)=$ width $A$ and $\langle i, j\rangle \in$ the indices of $A$. Then $(a \cdot A)_{i, j}=a \cdot A_{i, j}$.

(4) For all matrices $A, B$ over $\mathbb{R}$ of dimension $n$ holds $\operatorname{len}(A \cdot B)=\operatorname{len} A$ and $\operatorname{width}(A \cdot B)=$ width $B$ and $\operatorname{len}(A \cdot B)=n$ and $\operatorname{width}(A \cdot B)=n$.

(5) For every real number $a$ and for every matrix $A$ over $\mathbb{R}$ holds len $(a \cdot A)=$ len $A$ and $\operatorname{width}(a \cdot A)=$ width $A$.

\section{Calculation of Matrices}

We now state the proposition

(6) Let $A, B$ be matrices over $\mathbb{R}$. Suppose len $A=\operatorname{len} B$ and width $A=$ width $B$. Then $\operatorname{len}(A-B)=\operatorname{len} A$ and $\operatorname{width}(A-B)=$ width $A$ and for all $i, j$ such that $\langle i, j\rangle \in$ the indices of $A$ holds $(A-B)_{i, j}=A_{i, j}-B_{i, j}$.

Let us consider $n$ and let $A, B$ be matrices over $\mathbb{R}$ of dimension $n$. Then $A \cdot B$ is a matrix over $\mathbb{R}$ of dimension $n$.

The following propositions are true:

(7) For all matrices $A, B$ over $\mathbb{R}$ such that len $A=\operatorname{len} B$ and width $A=$ width $B$ and len $A>0$ holds $A+(B-B)=A$.

(8) For all matrices $A, B$ over $\mathbb{R}$ such that len $A=\operatorname{len} B$ and width $A=$ width $B$ and len $A>0$ holds $(A+B)-B=A$.

(9) For every matrix $A$ over $\mathbb{R}$ holds $(-1) \cdot A=-A$.

(10) For every matrix $A$ over $\mathbb{R}$ and for all elements $i, j$ of $\mathbb{N}$ such that $\langle i$, $j\rangle \in$ the indices of $A$ holds $(-A)_{i, j}=-A_{i, j}$.

(11) For all real numbers $a, b$ and for every matrix $A$ over $\mathbb{R}$ holds $(a \cdot b) \cdot A=$ $a \cdot(b \cdot A)$.

(12) For all real numbers $a, b$ and for every matrix $A$ over $\mathbb{R}$ holds $(a+b) \cdot A=$ $a \cdot A+b \cdot A$.

(13) For all real numbers $a, b$ and for every matrix $A$ over $\mathbb{R}$ holds $(a-b) \cdot A=$ $a \cdot A-b \cdot A$.

(14) For every matrix $A$ over $K$ such that $n>0$ and len $A>0$ holds $\left(\begin{array}{ccc}0 & \ldots & 0 \\ \vdots & \ddots & \vdots \\ 0 & \ldots & 0\end{array}\right)_{K}^{n \times(\operatorname{len} A)} \cdot A=\left(\begin{array}{ccc}0 & \ldots & 0 \\ \vdots & \ddots & \vdots \\ 0 & \ldots & 0\end{array}\right)_{K}^{n \times(\text { width } A)}$

(15) For all matrices $A, C$ over $K$ such that len $A=$ width $C$ and $\operatorname{len} C>0$ and len $A>0$ holds $(-C) \cdot A=-C \cdot A$.

(16) For all matrices $A, B, C$ over $K$ such that len $B=\operatorname{len} C$ and width $B=$ width $C$ and len $A=$ width $B$ and len $B>0$ and len $A>0$ holds $(B-C)$. $A=B \cdot A-C \cdot A$. 
(17) For all matrices $A, B, C$ over $\mathbb{R}$ such that len $A=\operatorname{len} B$ and width $A=$ width $B$ and len $C=$ width $A$ and len $A>0$ and len $C>0$ holds $(A-B)$. $C=A \cdot C-B \cdot C$.

(18) For every element $m$ of $\mathbb{N}$ and for all matrices $A, C$ over $K$ such that width $A>0$ and len $A>0$ holds $A \cdot\left(\begin{array}{ccc}0 & \ldots & 0 \\ \vdots & \ddots & \vdots \\ 0 & \ldots & 0\end{array}\right)_{K}^{(\text {width } A) \times m}=$ $\left(\begin{array}{ccc}0 & \ldots & 0 \\ \vdots & \ddots & \vdots \\ 0 & \cdots & 0\end{array}\right)_{K}^{(\operatorname{len} A) \times m}$

(19) For all matrices $A, C$ over $K$ such that width $A=\operatorname{len} C$ and len $A>0$ and len $C>0$ holds $A \cdot-C=-A \cdot C$.

(20) For all matrices $A, B, C$ over $K$ such that len $B=\operatorname{len} C$ and width $B=$ width $C$ and len $B=$ width $A$ and len $B>0$ and len $A>0$ holds $A \cdot(B-$ $C)=A \cdot B-A \cdot C$.

(21) For all matrices $A, B, C$ over $\mathbb{R}$ such that len $A=\operatorname{len} B$ and width $A=$ width $B$ and width $C=$ len $A$ and len $C>0$ and len $A>0$ holds $C \cdot(A-$ $B)=C \cdot A-C \cdot B$.

(22) Let $A, B, C$ be matrices over $\mathbb{R}$. Suppose that

(i) $\operatorname{len} A=\operatorname{len} B$,

(ii) width $A=$ width $B$,

(iii) $\operatorname{len} C=\operatorname{len} A$,

(iv) width $C=$ width $A$, and

(v) for all elements $i, j$ of $\mathbb{N}$ such that $\langle i, j\rangle \in$ the indices of $A$ holds $C_{i, j}=A_{i, j}-B_{i, j}$.

Then $C=A-B$.

(23) For all finite sequences $x_{1}, x_{2}$ of elements of $\mathbb{R}$ such that len $x_{1}=$ len $x_{2}$ and len $x_{1}>0$ holds LineVec2Mx $\left(x_{1}-x_{2}\right)=\operatorname{LineVec} 2 \mathrm{Mx} x_{1}-$ LineVec2Mx $x_{2}$.

(24) For all finite sequences $x_{1}, x_{2}$ of elements of $\mathbb{R}$ such that len $x_{1}=$ len $x_{2}$ and len $x_{1}>0$ holds ColVec $2 \mathrm{Mx}\left(x_{1}-x_{2}\right)=\operatorname{ColVec} 2 \mathrm{Mx} x_{1}-$ ColVec2Mx $x_{2}$.

(25) Let $A, B$ be matrices over $\mathbb{R}$. Suppose len $A=\operatorname{len} B$ and width $A=$ width $B$. Let $i$ be a natural number. If $1 \leq i$ and $i \leq \operatorname{len} A$, then Line $(A-$ $B, i)=\operatorname{Line}(A, i)-\operatorname{Line}(B, i)$.

(26) Let $A, B$ be matrices over $\mathbb{R}$. Suppose len $A=\operatorname{len} B$ and width $A=$ width $B$. Let $i$ be a natural number. If $1 \leq i$ and $i \leq$ width $A$, then $(A-B)_{\square, i}=A_{\square, i}-B_{\square, i}$.

(27) Let $A$ be a matrix over $\mathbb{R}$ of dimension $n \times k, B$ be a matrix over $\mathbb{R}$ of 
dimension $k \times m$, and $C$ be a matrix over $\mathbb{R}$ of dimension $m \times l$. If $n>0$ and $k>0$ and $m>0$, then $(A \cdot B) \cdot C=A \cdot(B \cdot C)$.

(28) For all matrices $A, B, C$ over $\mathbb{R}$ of dimension $n$ holds $(A \cdot B) \cdot C=$ $A \cdot(B \cdot C)$.

(29) For every matrix $A$ over $D$ of dimension $n$ holds $\left(A^{\mathrm{T}}\right)^{\mathrm{T}}=A$.

(30) For all matrices $A, B$ over $\mathbb{R}$ of dimension $n$ holds $(A \cdot B)^{\mathrm{T}}=B^{\mathrm{T}} \cdot A^{\mathrm{T}}$.

(31) For every matrix $A$ over $\mathbb{R}$ such that $n>0$ and len $A=n$ and width $A=$ $m$ holds $-A+A=\left(\begin{array}{ccc}0 & \ldots & 0 \\ \vdots & \ddots & \vdots \\ 0 & \ldots & 0\end{array}\right)_{\mathbb{R}}^{n \times m}$.

\section{Determinants}

Let us consider $n$ and let $A$ be a matrix over $\mathbb{R}$ of dimension $n$. Then $\left(\mathbb{R} \rightarrow \mathbb{R}_{\mathrm{F}}\right) A$ is a matrix over $\mathbb{R}_{\mathrm{F}}$ of dimension $n$.

Let us consider $n$ and let $A$ be a matrix over $\mathbb{R}$ of dimension $n$. The functor Det $A$ yielding a real number is defined as follows:

(Def. 1) $\operatorname{Det} A=\operatorname{Det}\left(\mathbb{R} \rightarrow \mathbb{R}_{\mathrm{F}}\right) A$.

We now state a number of propositions:

(32) For every matrix $A$ over $\mathbb{R}$ of dimension 2 holds $\operatorname{Det} A=A_{1,1} \cdot A_{2,2}$ $A_{1,2} \cdot A_{2,1}$.

(33) For all finite sequences $s_{1}, s_{2}, s_{3}$ such that len $s_{1}=n$ and len $s_{2}=n$ and len $s_{3}=n$ holds $\left\langle s_{1}, s_{2}, s_{3}\right\rangle$ is tabular.

(34) Let $p_{1}, p_{2}, p_{3}$ be finite sequences of elements of $D$. Suppose len $p_{1}=n$ and len $p_{2}=n$ and len $p_{3}=n$. Then $\left\langle p_{1}, p_{2}, p_{3}\right\rangle$ is a matrix over $D$ of dimension $3 \times n$.

(35) For all elements $a_{1}, a_{2}, a_{3}, b_{1}, b_{2}, b_{3}, c_{1}, c_{2}, c_{3}$ of $D$ holds $\left\langle\left\langle a_{1}, a_{2}, a_{3}\right\rangle\right.$, $\left.\left\langle b_{1}, b_{2}, b_{3}\right\rangle,\left\langle c_{1}, c_{2}, c_{3}\right\rangle\right\rangle$ is a matrix over $D$ of dimension 3 .

(36) Let $A$ be a matrix over $D$ of dimension $n, p$ be a finite sequence of elements of $D$, and $i$ be a natural number. If $p=A(i)$ and $i \in \operatorname{Seg} n$, then len $p=n$.

(37) For every matrix $A$ over $D$ of dimension 3 holds $A=\left\langle\left\langle A_{1,1}, A_{1,2}, A_{1,3}\right\rangle\right.$, $\left.\left\langle A_{2,1}, A_{2,2}, A_{2,3}\right\rangle,\left\langle A_{3,1}, A_{3,2}, A_{3,3}\right\rangle\right\rangle$.

(38) Let $A$ be a matrix over $\mathbb{R}$ of dimension 3. Then $\operatorname{Det} A=\left(\left(\left(A_{1,1} \cdot A_{2,2}\right.\right.\right.$. $\left.\left.A_{3,3}-A_{1,3} \cdot A_{2,2} \cdot A_{3,1}-A_{1,1} \cdot A_{2,3} \cdot A_{3,2}\right)+A_{1,2} \cdot A_{2,3} \cdot A_{3,1}\right)-A_{1,2} \cdot A_{2,1} \cdot$ $\left.A_{3,3}\right)+A_{1,3} \cdot A_{2,1} \cdot A_{3,2}$.

(39) For every permutation $f$ of $\operatorname{Seg} 0$ holds $f=\varepsilon_{\mathbb{N}}$.

(40) The permutations of 0-element set $=\left\{\varepsilon_{\mathbb{N}}\right\}$.

(41) For every matrix $A$ over $K$ of dimension 0 holds $\operatorname{Det} A=1_{K}$. 
(42) For every matrix $A$ over $\mathbb{R}$ of dimension 0 holds $\operatorname{Det} A=1$.

(43) For every natural number $n$ and for every matrix $A$ over $K$ of dimension $n$ holds $\operatorname{Det} A=\operatorname{Det}\left(A^{\mathrm{T}}\right)$.

(44) For every matrix $A$ over $\mathbb{R}$ of dimension $n$ holds $\operatorname{Det} A=\operatorname{Det}\left(A^{\mathrm{T}}\right)$.

(45) For all matrices $A, B$ over $K$ of dimension $n \operatorname{holds} \operatorname{Det}(A \cdot B)=\operatorname{Det} A$. Det $B$.

(46) For all matrices $A, B$ over $\mathbb{R}$ of dimension $n$ holds $\operatorname{Det}(A \cdot B)=\operatorname{Det} A$. Det $B$.

\section{Matrix as Operator}

We now state a number of propositions:

(47) Let $x, y$ be finite sequences of elements of $\mathbb{R}$ and $A$ be a matrix over $\mathbb{R}$. If len $x=\operatorname{len} A$ and len $y=\operatorname{len} x$ and len $x>0$ and len $A>0$, then $(x-y) \cdot A=x \cdot A-y \cdot A$.

(48) Let $x, y$ be finite sequences of elements of $\mathbb{R}$ and $A$ be a matrix over $\mathbb{R}$. If len $x=$ width $A$ and len $y=\operatorname{len} x$ and len $x>0$ and len $A>0$, then $A \cdot(x-y)=A \cdot x-A \cdot y$.

(49) Let $x$ be a finite sequence of elements of $\mathbb{R}$ and $A$ be a matrix over $\mathbb{R}$. If len $A=\operatorname{len} x$ and len $x>0$ and width $A>0$, then $(-x) \cdot A=-x \cdot A$.

(50) Let $x$ be a finite sequence of elements of $\mathbb{R}$ and $A$ be a matrix over $\mathbb{R}$. If len $x=$ width $A$ and len $A>0$ and len $x>0$, then $A \cdot-x=-A \cdot x$.

(51) Let $x$ be a finite sequence of elements of $\mathbb{R}$ and $A$ be a matrix over $\mathbb{R}$. If len $x=$ len $A$ and len $x>0$ and width $A>0$, then $x \cdot-A=-x \cdot A$.

(52) Let $x$ be a finite sequence of elements of $\mathbb{R}$ and $A$ be a matrix over $\mathbb{R}$. If len $x=$ width $A$ and len $A>0$ and len $x>0$, then $(-A) \cdot x=-A \cdot x$.

(53) Let $a$ be a real number, $x$ be a finite sequence of elements of $\mathbb{R}$, and $A$ be a matrix over $\mathbb{R}$. If width $A=\operatorname{len} x$ and len $x>0$ and len $A>0$, then $A \cdot(a \cdot x)=a \cdot(A \cdot x)$.

(54) Let $x$ be a finite sequence of elements of $\mathbb{R}$ and $A, B$ be matrices over $\mathbb{R}$. If len $x=$ len $A$ and len $A=\operatorname{len} B$ and width $A=$ width $B$ and len $A>0$, then $x \cdot(A-B)=x \cdot A-x \cdot B$.

(55) Let $x$ be a finite sequence of elements of $\mathbb{R}$ and $A, B$ be matrices over $\mathbb{R}$. If len $x=$ width $A$ and len $A=\operatorname{len} B$ and width $A=$ width $B$ and len $x>0$ and len $A>0$, then $(A-B) \cdot x=A \cdot x-B \cdot x$.

(56) For every finite sequence $x$ of elements of $\mathbb{R}$ and for every matrix $A$ over $\mathbb{R}$ such that len $A=\operatorname{len} x$ holds LineVec $2 \mathrm{Mx} x \cdot A=\operatorname{LineVec} 2 \mathrm{Mx}(x \cdot A)$.

(57) Let $x$ be a finite sequence of elements of $\mathbb{R}$ and $A, B$ be matrices over $\mathbb{R}$. If len $x=\operatorname{len} A$ and width $A=\operatorname{len} B$, then $x \cdot(A \cdot B)=(x \cdot A) \cdot B$. 
(58) Let $x$ be a finite sequence of elements of $\mathbb{R}$ and $A$ be a matrix over $\mathbb{R}$. If width $A=\operatorname{len} x$ and len $x>0$ and len $A>0$, then $A \cdot \operatorname{ColVec} 2 \mathrm{Mx} x=$ $\operatorname{ColVec} 2 \mathrm{Mx}(A \cdot x)$.

(59) Let $x$ be a finite sequence of elements of $\mathbb{R}$ and $A, B$ be matrices over $\mathbb{R}$. If len $x=$ width $B$ and width $A=\operatorname{len} B$ and len $x>0$ and len $B>0$, then $(A \cdot B) \cdot x=A \cdot(B \cdot x)$.

(60) Let $B$ be a matrix over $\mathbb{R}$ of dimension $n \times m$ and $A$ be a matrix over $\mathbb{R}$ of dimension $m \times k$. Suppose $n>0$. Let given $i, j$. If $\langle i, j\rangle \in$ the indices of $B \cdot A$, then $(B \cdot A)_{i, j}=(\operatorname{Line}(B, i) \cdot A)(j)$.

(61) Let $A, B$ be matrices over $\mathbb{R}$ of dimension $n$ and given $i, j$. If $\langle i, j\rangle \in$ the indices of $B \cdot A$, then $(B \cdot A)_{i, j}=(\operatorname{Line}(B, i) \cdot A)(j)$.

(62) Let $A, B$ be matrices over $\mathbb{R}$ of dimension $n$. Suppose $n>0$. Let given $i, j$. If $\langle i, j\rangle \in$ the indices of $A \cdot B$, then $(A \cdot B)_{i, j}=\left(A \cdot B_{\square, j}\right)(i)$.

\section{Identity And Zero of Matrix of $\mathbb{R}$}

Let $n$ be an element of $\mathbb{N}$. The functor $1_{\mathbb{R}} \operatorname{matrix}(n)$ yields a matrix over $\mathbb{R}$ of dimension $n$ and is defined as follows:

(Def. 2) $1_{\mathbb{R}} \operatorname{matrix}(n)=\left(\mathbb{R}_{\mathrm{F}} \rightarrow \mathbb{R}\right)\left(\left(\begin{array}{ccc}1 & & 0 \\ & \ddots & \\ 0 & & 1\end{array}\right)_{\mathbb{R}_{\mathrm{F}}}^{n \times n}\right)$.

One can prove the following propositions:

(63)(i) For every $i$ such that $\langle i, i\rangle \in$ the indices of $1_{\mathbb{R}} \operatorname{matrix}(n)$ holds $\left(1_{\mathbb{R}} \operatorname{matrix}(n)\right)_{i, i}=1$, and

(ii) for all $i, j$ such that $\langle i, j\rangle \in$ the indices of $1_{\mathbb{R}} \operatorname{matrix}(n)$ and $i \neq j$ holds $\left(1_{\mathbb{R}} \operatorname{matrix}(n)\right)_{i, j}=0$.

(64) $\quad\left(1_{\mathbb{R}} \operatorname{matrix}(n)\right)^{\mathrm{T}}=1_{\mathbb{R}} \operatorname{matrix}(n)$.

(65) For all elements $n, m$ of $\mathbb{N}$ such that $n>0$ holds $\left(\begin{array}{ccc}0 & \ldots & 0 \\ \vdots & \ddots & \vdots \\ 0 & \ldots & 0\end{array}\right)_{\mathbb{R}}^{n \times m}+$ $\left(\begin{array}{ccc}0 & \ldots & 0 \\ \vdots & \ddots & \vdots \\ 0 & \ldots & 0\end{array}\right)_{\mathbb{R}}^{n \times m}=\left(\begin{array}{ccc}0 & \ldots & 0 \\ \vdots & \ddots & \vdots \\ 0 & \ldots & 0\end{array}\right)_{\mathbb{R}}^{n \times m}$

(66) For every real number $a$ such that $n>0$ holds $a \cdot\left(\begin{array}{ccc}0 & \ldots & 0 \\ \vdots & \ddots & \vdots \\ 0 & \ldots & 0\end{array}\right)_{\mathbb{R}}^{n \times m}=$ 
$\left(\begin{array}{ccc}0 & \cdots & 0 \\ \vdots & \ddots & \vdots \\ 0 & \cdots & 0\end{array}\right)_{\mathbb{R}}^{n \times m}$

(67) For every field $K$ and for every matrix $A$ over $K$ holds $A$. $\left(\begin{array}{lll}1 & & 0 \\ & \ddots & \\ 0 & & 1\end{array}\right)_{K}^{\text {width } A \times \text { width } A}=A$.

(68) For every matrix $A$ over $K$ holds $\left(\begin{array}{lll}1 & & 0 \\ & \ddots & \\ 0 & & 1\end{array}\right)_{K}^{\operatorname{len} A \times \operatorname{len} A} \cdot A=A$.

(69) For every matrix $A$ over $\mathbb{R}$ holds if $n=$ width $A$, then $A \cdot 1_{\mathbb{R}}$ matrix $(n)=A$ and if $m=\operatorname{len} A$, then $1_{\mathbb{R}} \operatorname{matrix}(m) \cdot A=A$.

(70) For every matrix $A$ over $\mathbb{R}$ of dimension $n$ holds $1_{\mathbb{R}} \operatorname{matrix}(n) \cdot A=A$.

(71) For every matrix $A$ over $\mathbb{R}$ of dimension $n$ holds $A \cdot 1_{\mathbb{R}} \operatorname{matrix}(n)=A$.

(72) Det $1_{\mathbb{R}} \operatorname{matrix}(n)=1$.

Let $n$ be an element of $\mathbb{N}$. The functor $0_{\mathbb{R}} \operatorname{matrix}(n)$ yields a matrix over $\mathbb{R}$ of dimension $n$ and is defined by:

(Def. 3) $0_{\mathbb{R}} \operatorname{matrix}(n)=\left(\begin{array}{ccc}0 & \ldots & 0 \\ \vdots & \ddots & \vdots \\ 0 & \ldots & 0\end{array}\right)_{\mathbb{R}}^{n \times n}$

One can prove the following proposition

(73) If $n>0$, then Det $0_{\mathbb{R}} \operatorname{matrix}(n)=0$.

Let us consider $n$ and let us consider $i$. The base fin $\operatorname{seq}(n, i)$ yielding a finite sequence of elements of $\mathbb{R}$ is defined by:

(Def. 4) The base fin $\operatorname{seq}(n, i)=\operatorname{Replace}(n \mapsto(0$ qua element of $\mathbb{R}), i, 1)$.

We now state several propositions:

(74) len (the base fin $\operatorname{seq}(n, i))=n$.

(75) If $1 \leq i$ and $i \leq n$, then (the base fin $\operatorname{seq}(n, i))(i)=1$.

(76) If $1 \leq i$ and $i \leq n$ and $1 \leq j$ and $j \leq n$ and $i \neq j$, then (the base fin seq( $n, i))(j)=0$.

(77)(i) The base fin seq $(1,1)=\langle 1\rangle$,

(ii) the base fin $\operatorname{seq}(2,1)=\langle 1,0\rangle$,

(iii) the base fin $\operatorname{seq}(2,2)=\langle 0,1\rangle$,

(iv) the base fin $\operatorname{seq}(3,1)=\langle 1,0,0\rangle$,

(v) the base fin seq $(3,2)=\langle 0,1,0\rangle$, and

(vi) the base fin $\operatorname{seq}(3,3)=\langle 0,0,1\rangle$.

(78) If $1 \leq i$ and $i \leq n$, then $\left(1_{\mathbb{R}} \operatorname{matrix}(n)\right)(i)=$ the base fin $\operatorname{seq}(n, i)$. 


\section{INVERSE OF MATRIX}

Let $n$ be an element of $\mathbb{N}$ and let $A$ be a matrix over $\mathbb{R}$ of dimension $n$. We say that $A$ is invertible if and only if:

(Def. 5) There exists a matrix $B$ over $\mathbb{R}$ of dimension $n$ such that $B \cdot A=$ $1_{\mathbb{R}} \operatorname{matrix}(n)$ and $A \cdot B=1_{\mathbb{R}} \operatorname{matrix}(n)$.

Let $n$ be an element of $\mathbb{N}$ and let $A$ be a matrix over $\mathbb{R}$ of dimension $n$. Let us assume that $A$ is invertible. The functor Inv $A$ yields a matrix over $\mathbb{R}$ of dimension $n$ and is defined as follows:

(Def. 6) $\operatorname{Inv} A \cdot A=1_{\mathbb{R}} \operatorname{matrix}(n)$ and $A \cdot \operatorname{Inv} A=1_{\mathbb{R}} \operatorname{matrix}(n)$.

Let us consider $n$. Note that $1_{\mathbb{R}} \operatorname{matrix}(n)$ is invertible.

We now state a number of propositions:

(79) $\quad \operatorname{Inv} 1_{\mathbb{R}} \operatorname{matrix}(n)=1_{\mathbb{R}} \operatorname{matrix}(n)$.

(80) For all matrices $A, B_{1}, B_{2}$ over $\mathbb{R}$ of dimension $n$ such that $B_{1} \cdot A=$ $1_{\mathbb{R}} \operatorname{matrix}(n)$ and $A \cdot B_{2}=1_{\mathbb{R}} \operatorname{matrix}(n)$ holds $B_{1}=B_{2}$ and $A$ is invertible.

(81) For every matrix $A$ over $\mathbb{R}$ of dimension $n$ such that $A$ is invertible holds $\operatorname{Det} \operatorname{Inv} A=\operatorname{Det} A^{-1}$.

(82) For every matrix $A$ over $\mathbb{R}$ of dimension $n$ such that $A$ is invertible holds $\operatorname{Det} A \neq 0$.

(83) Let $A, B$ be matrices over $\mathbb{R}$ of dimension $n$. Suppose $A$ is invertible and $B$ is invertible. Then $A \cdot B$ is invertible and $\operatorname{Inv} A \cdot B=\operatorname{Inv} B \cdot \operatorname{Inv} A$.

(84) For every matrix $A$ over $\mathbb{R}$ of dimension $n$ such that $A$ is invertible holds $\operatorname{Inv} \operatorname{Inv} A=A$.

(85) $\quad 1_{\mathbb{R}} \operatorname{matrix}(0)=0_{\mathbb{R}} \operatorname{matrix}(0)$ and $1_{\mathbb{R}} \operatorname{matrix}(0)=\emptyset$.

(86) For every finite sequence $x$ of elements of $\mathbb{R}$ such that len $x=n$ and $n>0$ holds $1_{\mathbb{R}} \operatorname{matrix}(n) \cdot x=x$.

(87) Let $n$ be an element of $\mathbb{N}, x, y$ be finite sequences of elements of $\mathbb{R}$, and $A$ be a matrix over $\mathbb{R}$ of dimension $n$. Suppose $A$ is invertible and len $x=n$ and len $y=n$ and $n>0$. Then $A \cdot x=y$ if and only if $x=\operatorname{Inv} A \cdot y$.

(88) For every finite sequence $x$ of elements of $\mathbb{R}$ such that len $x=n$ holds $x \cdot 1_{\mathbb{R}} \operatorname{matrix}(n)=x$.

(89) Let $x, y$ be finite sequences of elements of $\mathbb{R}$ and $A$ be a matrix over $\mathbb{R}$ of dimension $n$. Suppose $A$ is invertible and len $x=n$ and len $y=n$. Then $x \cdot A=y$ if and only if $x=y \cdot \operatorname{Inv} A$.

(90) Let $A$ be a matrix over $\mathbb{R}$ of dimension $n$. Suppose $n>0$ and $A$ is invertible. Let $y$ be a finite sequence of elements of $\mathbb{R}$. Suppose len $y=n$. Then there exists a finite sequence $x$ of elements of $\mathbb{R}$ such that len $x=n$ and $A \cdot x=y$. 
(91) Let $A$ be a matrix over $\mathbb{R}$ of dimension $n$. Suppose $A$ is invertible. Let $y$ be a finite sequence of elements of $\mathbb{R}$. Suppose len $y=n$. Then there exists a finite sequence $x$ of elements of $\mathbb{R}$ such that len $x=n$ and $x \cdot A=y$.

(92) Let $A$ be a matrix over $\mathbb{R}$ of dimension $n$ and $x, y$ be finite sequences of elements of $\mathbb{R}$. Suppose len $x=n$ and len $y=n$ and $x \cdot A=y$. Let $j$ be an element of $\mathbb{N}$. If $1 \leq j$ and $j \leq n$, then $y(j)=\left|\left(x, A_{\square, j}\right)\right|$.

(93) Let $A$ be a matrix over $\mathbb{R}$ of dimension $n$. Suppose that for every finite sequence $y$ of elements of $\mathbb{R}$ such that len $y=n$ there exists a finite sequence $x$ of elements of $\mathbb{R}$ such that len $x=n$ and $x \cdot A=y$. Then there exists a matrix $B$ over $\mathbb{R}$ of dimension $n$ such that $B \cdot A=1_{\mathbb{R}} \operatorname{matrix}(n)$.

(94) Let $x$ be a finite sequence of elements of $\mathbb{R}$ and $A$ be a matrix over $\mathbb{R}$ of dimension $n$. If $n>0$ and len $x=n$, then $A^{\mathrm{T}} \cdot x=x \cdot A$.

(95) Let $x$ be a finite sequence of elements of $\mathbb{R}$ and $A$ be a matrix over $\mathbb{R}$ of dimension $n$. If $n>0$ and len $x=n$, then $x \cdot A^{\mathrm{T}}=A \cdot x$.

(96) Let $A$ be a matrix over $\mathbb{R}$ of dimension $n$. Suppose that

(i) $n>0$, and

(ii) for every finite sequence $y$ of elements of $\mathbb{R}$ such that len $y=n$ there exists a finite sequence $x$ of elements of $\mathbb{R}$ such that len $x=n$ and $A \cdot x=y$. Then there exists a matrix $B$ over $\mathbb{R}$ of dimension $n$ such that $A \cdot B=$ $1_{\mathbb{R}} \operatorname{matrix}(n)$.

(97) Let $A$ be a matrix over $\mathbb{R}$ of dimension $n$. Suppose that

(i) $n>0$, and

(ii) for every finite sequence $y$ of elements of $\mathbb{R}$ such that len $y=n$ there exist finite sequences $x_{1}, x_{2}$ of elements of $\mathbb{R}$ such that len $x_{1}=n$ and len $x_{2}=n$ and $A \cdot x_{1}=y$ and $x_{2} \cdot A=y$.

Then $A$ is invertible.

\section{REFERENCES}

[1] Kanchun and Yatsuka Nakamura. The inner product of finite sequences and of points of $n$-dimensional topological space. Formalized Mathematics, 11(2):179-183, 2003.

[2] Grzegorz Bancerek. The ordinal numbers. Formalized Mathematics, 1(1):91-96, 1990.

[3] Grzegorz Bancerek and Krzysztof Hryniewiecki. Segments of natural numbers and finite sequences. Formalized Mathematics, 1(1):107-114, 1990.

[4] Czesław Byliński. Binary operations. Formalized Mathematics, 1(1):175-180, 1990.

[5] Czesław Byliński. Binary operations applied to finite sequences. Formalized Mathematics, 1(4):643-649, 1990

[6] Czesław Byliński. Finite sequences and tuples of elements of a non-empty sets. Formalized Mathematics, 1(3):529-536, 1990.

[7] Czesław Byliński. Functions and their basic properties. Formalized Mathematics, 1(1):5565, 1990.

[8] Czesław Byliński. Functions from a set to a set. Formalized Mathematics, 1(1):153-164, 1990.

[9] Czesław Byliński. Some basic properties of sets. Formalized Mathematics, 1(1):47-53, 1990.

[10] Czesław Byliński. The sum and product of finite sequences of real numbers. Formalized Mathematics, 1(4):661-668, 1990. 
[11] Agata Darmochwal. The Euclidean space. Formalized Mathematics, 2(4):599-603, 1991.

[12] Shigeru Furuya. Matrix and Determinant. Baifuukan (in Japanese), 1957.

[13] Felix R. Gantmacher. The Theory of Matrices. AMS Chelsea Publishing, 1959.

[14] Katarzyna Jankowska. Matrices. Abelian group of matrices. Formalized Mathematics, 2(4):475-480, 1991.

[15] Katarzyna Jankowska. Transpose matrices and groups of permutations. Formalized Mathematics, 2(5):711-717, 1991.

[16] Jarosław Kotowicz. Real sequences and basic operations on them. Formalized Mathematics, 1(2):269-272, 1990.

[17] Eugeniusz Kusak, Wojciech Leończuk, and Michał Muzalewski. Abelian groups, fields and vector spaces. Formalized Mathematics, 1(2):335-342, 1990.

[18] Yatsuka Nakamura. Determinant of some matrices of field elements. Formalized Mathematics, 14(1):1-5, 2006.

[19] Yatsuka Nakamura, Nobuyuki Tamaura, and Wenpai Chang. A theory of matrices of real elements. Formalized Mathematics, 14(1):21-28, 2006.

[20] Yatsuka Nakamura and Hiroshi Yamazaki. Calculation of matrices of field elements. Part I. Formalized Mathematics, 11(4):385-391, 2003.

[21] Library Committee of the Association of Mizar Users. Binary operations on numbers. To appear in Formalized Mathematics.

[22] Andrzej Trybulec. Subsets of complex numbers. To appear in Formalized Mathematics.

[23] Andrzej Trybulec. Binary operations applied to functions. Formalized Mathematics, 1(2):329-334, 1990.

[24] Andrzej Trybulec. Tarski Grothendieck set theory. Formalized Mathematics, 1(1):9-11,

[25] Andrzej Trybulec and Agata Darmochwał. Boolean domains. Formalized Mathematics, $1(\mathbf{1}): 187-190,1990$.

[26] Wojciech A. Trybulec. Binary operations on finite sequences. Formalized Mathematics, 1(5):979-981, 1990.

[27] Wojciech A. Trybulec. Groups. Formalized Mathematics, 1(5):821-827, 1990.

[28] Wojciech A. Trybulec. Pigeon hole principle. Formalized Mathematics, 1(3):575-579, 1990.

[29] Wojciech A. Trybulec. Vectors in real linear space. Formalized Mathematics, 1(2):291$296,1990$.

[30] Zinaida Trybulec. Properties of subsets. Formalized Mathematics, 1(1):67-71, 1990.

[31] Edmund Woronowicz. Relations and their basic properties. Formalized Mathematics, 1(1):73-83, 1990.

[32] Hiroshi Yamazaki, Yoshinori Fujisawa, and Yatsuka Nakamura. On replace function and swap function for finite sequences. Formalized Mathematics, 9(3):471-474, 2001.

[33] Xiaopeng Yue, Xiquan Liang, and Zhongpin Sun. Some properties of some special matrices. Formalized Mathematics, 13(4):541-547, 2005.

[34] Katarzyna Zawadzka. The product and the determinant of matrices with entries in a field. Formalized Mathematics, 4(1):1-8, 1993.

[35] Bo Zhang and Yatsuka Nakamura. The definition of finite sequences and matrices of probability, and addition of matrices of real elements. Formalized Mathematics, 14(3):101108,2006 . 